\title{
RIZOBACTERIAS PROMOTORAS DE CRECIMIENTO VEGETAL (PGPR): UNA REVISIÓN SISTEMÁTICA 1990-2019
}

\author{
PLANT GROWTH PROMOTING RHIZOBACTERIA (PGPR): \\ A SYSTEMATIC REVIEW 1990-2019
}

\section{${ }^{1}$ Anny Melissa Posada Castaño, ${ }^{2}$ Diana Patricia Mejía Durango, ${ }^{3}$ Diana Polanco-Echeverry, ${ }^{4}$ Jaiberth Antonio Cardona Arias}

1 Microbióloga Industrial y Ambiental, MSc (estudiante) en Microbiología Universidad de Antioquia, Medellín, Colombia. 2 Microbióloga Industrial y Ambiental, Universidad de Antioquia, Medellín, Colombia.

${ }^{3}$ PhD Agroecología, Universidad de Antioquia, Medellín, Colombia.

${ }^{4}$ MSc Epidemiología, MSc Economía Aplicada, PhD (candidato) Salud Pública, Universidad de Antioquia, Medellín, Colombia.

${ }^{1}$ anny.posada@udea.edu.co, ${ }^{2}$ diana.mejiad@udea.edu.co,

${ }^{3}$ diana.polanco@udea.edu.co, ${ }^{4}$ jaiberth.cardona@udea.edu.co

Citación: Posada, A., Mejía, D., Polanco-Echeverry, D., y Cardona, J. (2021). Rizobacterias promotoras de crecimiento vegetal (PGPR): Una revisión sistemática 1990-2019. Revista de Investigación Agraria y Ambiental, $12(2), 161$ - 178. DOI: https://doi.org/10.22490/21456453.4040

\section{RESUMEN}

Contextualización: actualmente se utilizan grandes cantidades de agroquímicos para aumentar la productividad agrícola, lo cual ha acarreado múltiples problemáticas ambientales que podrían subsanarse con el uso de rizobacterias promotoras de crecimiento vegetal (Plant Growth Promoting Rhizobacteria, PGPR), fomentando el desarrollo de una agricultura sostenible.

Vacío de conocimiento: la información se encuentra dispersa $y$, a la fecha, no existe un análisis sistemático que permita una descripción precisa de las investigaciones sobre PGPR, lo cual representa una limitación para su aplicación y el desarrollo de investigaciones experimentales posteriores.
Objetivos: describir las principales aplicaciones de las PGPR en investigaciones originales a nivel mundial.

Metodología: la revisión sistemática se realizó en tres bases de datos multidisciplinarias siguiendo las recomendaciones Cochrane y PRISMA. Se garantizó exhaustividad, reproducibilidad y evaluación de la calidad. Los análisis se basaron en frecuencias e intervalos de confianza del $95 \%$ para las proporciones.

Resultados y conclusiones: se identificaron 2.525 estudios y solo 160 cumplieron el protocolo de selección. La mayoría de los estudios proceden de la India; los principales géneros microbianos descritos fueron Bacillus, Pseudomonas 
y Enterobacter; y los mecanismos PGPR más investigados fueron: producción de ácido indolacético (AIA), solubilización de fosfato, antagonismo y producción de sideróforos. Las especies vegetales más frecuentes en los estudios fueron maíz, tomate, trigo y arroz, en las cuales los principales efectos de las PGPR se relacionaron con el peso y la longitud de la raíz y de la planta en general. Se concluye que esta revisión identificó los géneros de PGPR, mecanismos de acción PGPR y las especies vegetales de mayor frecuencia en los estudios, variables útiles para resumir la evidencia en este campo del conocimiento e identificar las principales líneas de acción en futuras investigaciones tendientes a mejorar la salud, producción de cultivos y sostenibilidad ambiental.

Palabras clave: agricultura sostenible; cultivos; microorganismos benéficos; disponibilidad de nutrientes; biocontrol; PGPR; sistematización de estudios

\section{ABSTRACT}

Contextualization: nowadays, large quantities of agrochemicals are used to increase agricultural productivity; this has caused many environmental problems that can be solved using Plant Growth-Promoting Rhizobacteria (PGPR), encouraging sustainable agriculture.

Knowledge gap: information is often scattered and, to date, there is no systematic analysis that allows an accurate description of studies about PGPR, which represents a limitation in further experimental research developments.
Purpose: describe the main applications of PGPR in original research worldwide.

Methodology: the systematic review was carried out in three multidisciplinary databases following the Cochrane and PRISMA recommendations. Completeness, reproducibility and quality assessment were guaranteed. The analyzes were based on frequencies and 95\% confidence intervals for the proportions

Results and conclusions: 2,525 studies were identified and only 160 met the selection protocol. The majority of studies are from India; the main microbial genera described were Bacillus, Pseudomonas and Enterobacter; and the most investigated PGPR mechanisms were indolacetic acid production (AIA), phosphate solubilization, antagonism and siderophores production. The most frequent plant species in the studies were corn, tomato, wheat and rice, in which the main effects of the PGPR were related weight and length of the root and the plant in general. It is concluded that this review identified the genres of PGPR, mechanisms of action PGPR and plant species of greater frequency in the studies, useful variables to summarize the evidence in this field and identify the main lines of action in future research aimed at improving health, crop production and environmental sustainability.

Keywords: sustainable agriculture; crops; beneficial microorganisms; biocontrol; nutrient availability; PGPR; studies systematization 


\section{RESUMEN GRÁFICO}
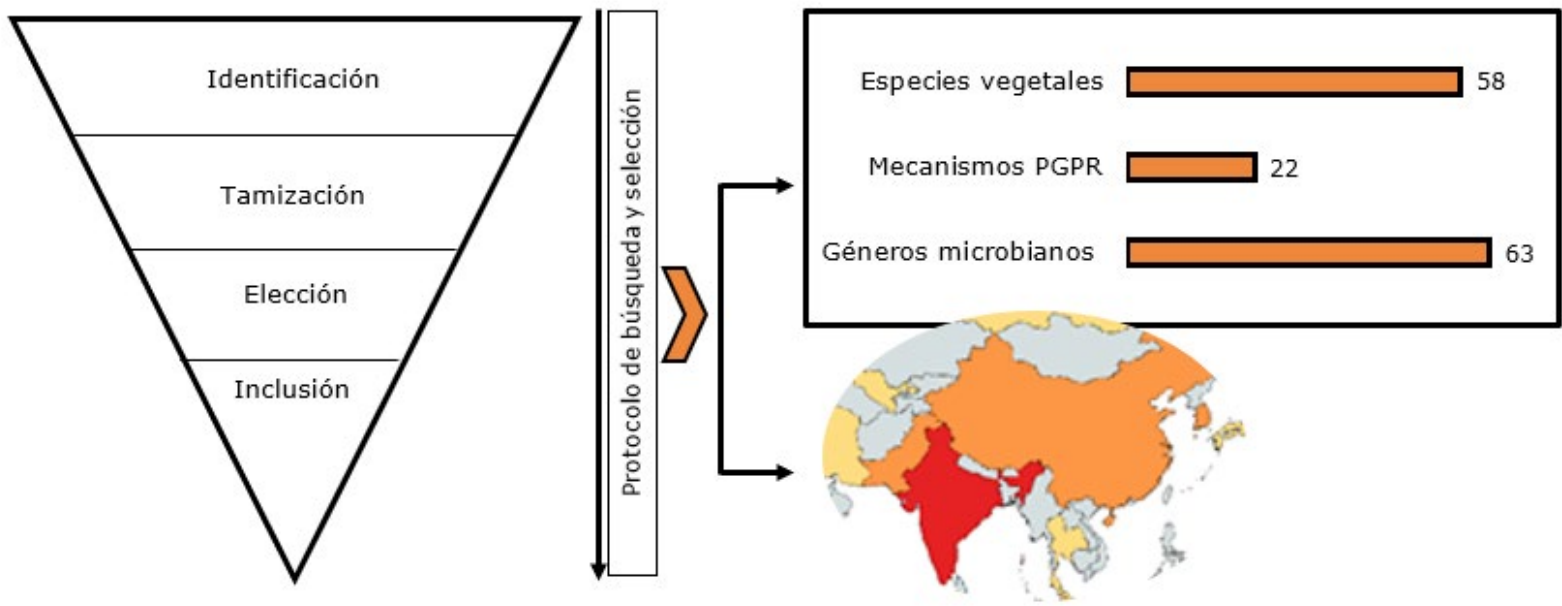

Fuente: autores

\section{INTRODUCCIÓN}

En la actualidad, la agricultura intensiva se encuentra asociada con múltiples problemáticas ambientales que incluyen enfermedades, sequía, deficiencia de nutrientes, erosión, pérdidas de la biodiversidad, y fragmentación del paisaje, entre otros. Además, el uso masivo de compuestos químicos conlleva un incremento de los costos de producción para los agricultores de todo el mundo y conduce a una importante contaminación ambiental; así como a la disminución a largo plazo de la productividad de los cultivos (Jewell et al., 2010).

En este contexto, las prácticas agrícolas se deben dirigir a una agricultura sostenible con el fin de mantener el rendimiento de los cultivos y preservar los agroecosistemas. Una de las alternativas para lograr la sostenibilidad en la producción agrícola es el uso de microorganismos del suelo, como las rizobacterias promotoras de crecimiento vegetal (PGPR, Plant Growth Promoting Rhizobacteria), que colonizan activamente las rizósfera, ejerciendo un efecto benéfico sobre el desarrollo de las plantas. Las PGPR pueden influir en el crecimiento de las plantas mediante mecanismos directos como la fijación de nitrógeno, la solubilización de minerales y la producción o cambio en la concentración de hormonas vegetales; y por mecanismos indirectos como producción de metabolitos antifúngicos, sideróforos, actividad lítica, inducción de resistencia sistémica, competencia y desplazamiento (Schroth y Becker, 1990; Kloepper, 1993).

Por lo anterior, en las últimas décadas se ha incrementado el interés por el estudio de este grupo de bacterias, no solo por su potencial para la estimulación del crecimiento de cultivos o como agentes de biocontrol, sino además por su uso prometedor en la descontaminación de suelos, reforestación y recuperación de ecosistemas. Es por esto que se ha hecho necesario realizar estudios experimentales que permitan evaluar el papel de las PGPRs en la agricultura. Mukhtar et al. (2017) demostraron el efecto de bacterias solubilizadoras en el crecimiento del trigo; Xiang et al. (2017) se centraron en la evaluación del potencial de las PGPR como 
agentes de biocontrol en soja. También se ha demostrado la influencia de las PGPRs para estimular el crecimiento de las plantas en diferentes condiciones de estrés, como lo presentan Mahmood et al. (2016) bajo condiciones de salinidad. De manera similar, Rizvi y Khan (2018) evaluaron el potencial PGPR bajo condiciones de estrés por metales. También se han llevado a cabo diversas revisiones narrativas, por ejemplo, Etesami y Maheshwari (2018) describen los mecanismos de acción a través de los cuales las PGPR podrían aliviar el estrés abiótico en las plantas, mientras que la revisión de Mhatre et al. (2018) se concentró en las estrategias de control biológico de nematodos basados en PGPR.

Estos antecedentes evidencian la disponibilidad de investigaciones sobre las PGPR; sin embargo, la información se encuentra dispersa y, a la fecha, no existe un análisis sistemático y exhaustivo que describa y sintetice de forma global las investigaciones sobre PGPR, así como los mecanismos de acción y su efecto sobre diferentes cultivos, lo cual representa una limitación en este campo del conocimiento y una barrera para el desarrollo de investigaciones experimentales posteriores. Por lo anterior, el objetivo de este estudio fue realizar un análisis sistemático de las publicaciones sobre PGPR en el ámbito mundial, permitiendo sintetizar la evidencia de los mecanismos de acción, variables de respuesta de las plantas, microorganismos y plantas utilizadas en la evaluación del potencial PGPR.

\section{MATERIALES Y MÉTODOS}

Tipo de estudio: revisión sistemática de la literatura científica, bajo el enfoque amplio de Cochrane, que corresponde a un resumen completo de la evidencia. Este enfoque es una aproximación práctica para resumir la literatura científica mundial disponible en un campo específico del conocimiento, actualizar la evidencia alrededor de una temática en particular, agrupar las investigaciones disponibles, analizar las tendencias, y generar hipótesis o identificar áreas relevantes para orientar estudios posteriores (Centro Cochrane Iberoamericano, 2012).

\section{Protocolo de investigación de acuerdo con PRISMA (Urrutia y Bonfill, 2010)}

Identificación de artículos y estrategia de búsqueda: se realizó una búsqueda sistemática de literatura en las bases de datos ScienceDirect, PubMed y Scielo. En ScienceDirect se empleó la opción "Título/Resumen/Palabras claves" de la búsqueda avanzada, ingresando el término "PGPR" y "Plant growth promoting rhizobacteria". En PubMed y Scielo se usó la opción "Título/Resumen" de la búsqueda, ingresando los mismos términos. Se exportaron las referencias encontradas, junto con su respectivo resumen, a un administrador de referencias Zotero, en el que se eliminaron las referencias duplicadas. De manera retrospectiva, no se aplicaron restricciones $y$, de manera prospectiva, la última actualización del protocolo se realizó en julio del 2019, de esta forma se estableció la ventana de tiempo de esta revisión entre 1990, que corresponde a la década en que se halló la publicación más antigua, y el 2018, con una actualización manual para identificar nuevas publicaciones en 2019.

Tamizado: se incluyeron investigaciones con los términos de búsqueda en título, resumen o palabras clave, investigaciones originales (con 
lo cual se excluyeron editoriales y revisiones de tema) y estudios en los cuales el desenlace central se enfocaba en rizobacterias promotoras de crecimiento vegetal.

Elección: en esta fase se excluyeron los artículos en los que no fue explícita la evaluación del mecanismo promotor de crecimiento vegetal por parte de microrganismos; que no realizaran ensayos in vitro; y que incluyeran pruebas de estimulación in vivo bajo condiciones no inducidas, es decir, se excluyeron aquellos estudios donde realizaron pruebas in vivo bajo condiciones ambientales controladas (cámara de crecimiento), esto con el fin de describir el efecto directo de las PGPRs sobre el crecimiento de las plantas, sin el control de factores ambientales sujetos a las pruebas experimentales. De igual manera, se excluyeron los artículos que no clasificaran de manera taxonómica los microorganismos evaluados mediante técnicas moleculares, y estudios que evaluaran microorganismos genéticamente modificados, de forma que se sistematizara las publicaciones sobre PGPR en condiciones naturales y experimentalmente reproducibles.

Inclusión: se incluyeron artículos originales de investigación reportados en la literatura científica comprendidos en los años 19902018 extrayendo las variables de título, autores, año, país, mecanismo de las PGPR, tipo de planta y sus parámetros de crecimiento, así como el microorganismo evaluado. Se extrajeron los datos de cada publicación y se tabularon en un archivo plano estandarizado en Excel.

Reproducibilidad de la revisión sistemática: la evaluación de la reproducibilidad del estudio se llevó a cabo mediante la implementación de todo el proceso de búsqueda y selección de artículos, así como la recopilación de información, por dos revisores independientes, y el desacuerdo se resolvió por consenso o verificación por un tercero.

Análisis de la información: con las variables del estudio, se realizó una síntesis cualitativa de la información mediante frecuencias absolutas (n) y relativas (\%), así como por intervalos de confianza del $95 \%$ para las proporciones mediante el estadístico $\mathrm{P} \pm\{\mathrm{Za} / 2 *[\sqrt{ }(\mathrm{PQ} / \mathrm{n})]\}$, donde $\mathrm{P}$ es la proporción, $\mathrm{Za} / 2$ el nivel de confianza (del 95\%), Q es el complemento de $\mathrm{P}$ y $\mathrm{n}$ el número de datos analizados (o la frecuencia absoluta). Para describir la distribución de los estudios según el país de origen, se realizó un mapa empleando la plataforma MapChart Create Custom Map, y tomando tres rangos, el primero incluyó los países con 1-10 artículos, el segundo era para países con 11-20 estudios y el tercero abarca los países con más de 20 manuscritos.

\section{RESULTADOS Y DISCUSIÓN Descripción de los artículos incluidos}

Se identificaron 2,525 artículos de investigación de los cuales se tamizaron con lectura de títulos y resumen 1,570 , de estos solo 160 cumplieron con el protocolo de esta revisión (Figura 1). 


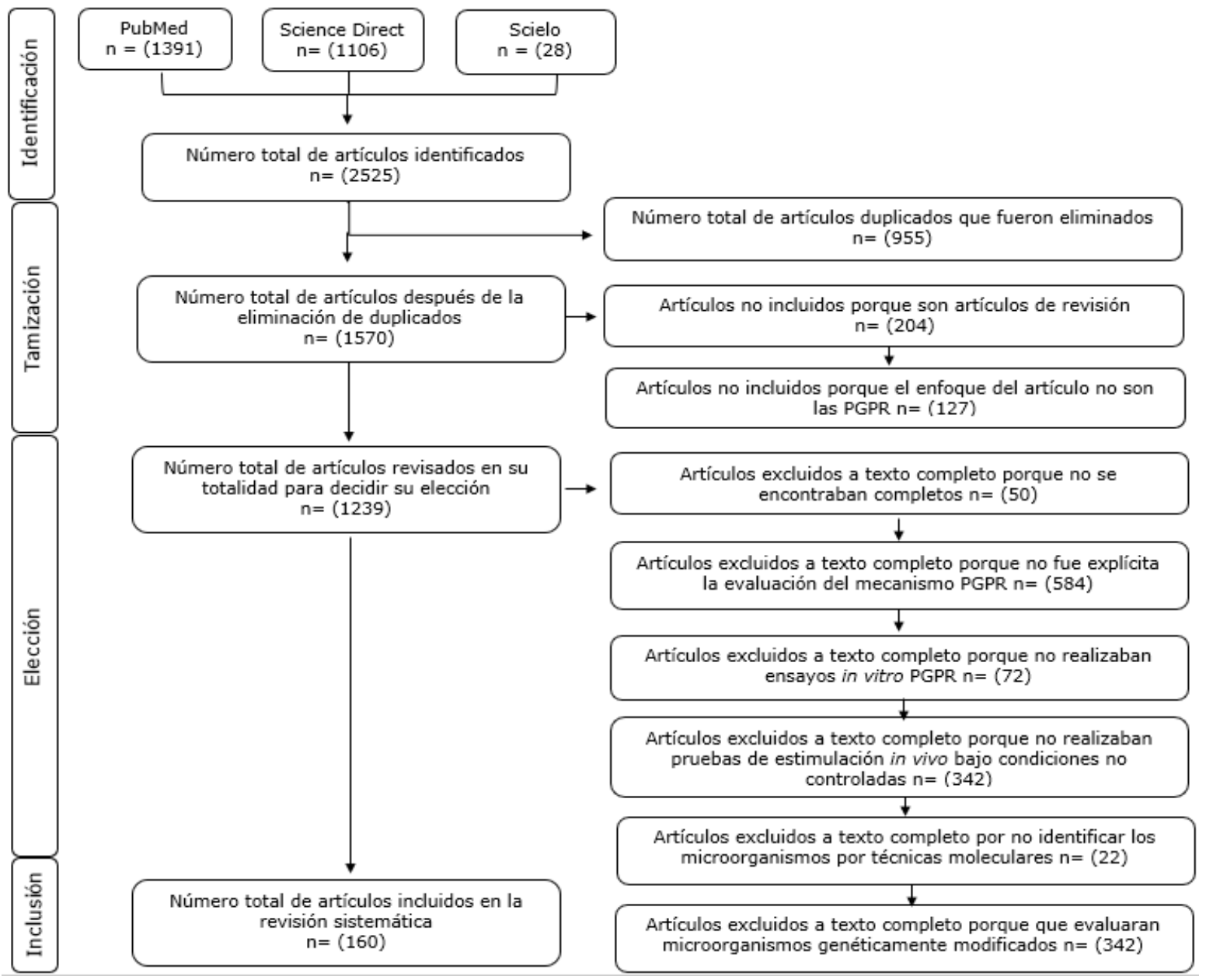

Figura 1. Flujograma de búsqueda y selección de los estudios incluidos en esta revisión acorde con las cuatro fases de la guía PRISMA.

Fuente: Autores.

De los 160 estudios (ver Anexo), el 74.4\% $(n=119)$ corresponde a investigaciones realizadas en Asia; $18.1 \%(n=29)$ en América; $5.6 \%$ $(n=9)$ en Europa; y $1.9 \%(n=3)$ en África, siendo India $(n=58)$, China $(n=18)$ y Corea del sur $(n=15)$ los países con el mayor número de investigaciones (Figura 2 ).

Lo anterior evidenció que la mayoría de estudios proceden de Asia, principalmente de la India, esto podría relacionarse de manera indirecta con el hecho de que este país, sumado a Estados Unidos, son potencias agrícolas mundiales. Aunque el país asiático presenta mayor investigación en agroecología, al tiempo que se encuentra entre los principales productores de varios cultivos, contando con la mayor área cultivada de trigo, arroz y algodón; además, es el segundo mayor productor de arroz, trigo, algodón, caña de azúcar, frutas, verduras y té (World Bank, 2012; Deshpande, 2017). También se debe destacar que el arroz es el principal cultivo alimentario no solo en India sino también en el mundo, resaltando además que el $90 \%$ del arroz mundial se produce en Asia y según estadísticas de Agriculture Market Information System (AMIS) en el 2018, India produjo 115.63 millones de toneladas de arroz. Por lo anterior, es plausible afirmar que en este país se hace una búsqueda de nuevas técnicas agrícolas amigables con el ambiente que generen a su vez un incremento en el rendimiento de los cultivos. Esto se evidenció en el estudio de Wezel y Soldat (2009), donde demostraron 
que India se encuentra dentro de los países con más publicaciones sobre agroecología, incrementando el interés en el desarrollo de diferentes tecnología que involucra el aislamiento y evaluación de microorganismos benéficos para las plantas como las PGPR (Lavakush et al., 2014; Karthiba et al., 2010).

\section{Investigación sobre PGPR en América}

Si bien India es el país con mayor número de investigaciones sobre PGPR según protocolo de búsqueda y selección, cabe resaltar que existen otros países latinoamericanos que también han contribuido en el estudio de las PGPR, aunque en menor medida. Se encontró que, en Colombia, las investigaciones obtenidas se encontraban enfocadas en la bioprospección de PGPR, la evaluación de sus mecanismos de acción, así como su efecto sobre el crecimiento vegetal. En tal sentido, Posada et al. (2016) exploraron una estrategia de bioprospección de bacterias aerobias formadoras de endospora (BAFE) que podrían ser útiles para desarrollar un producto biotecnológico para promover el crecimiento de las plantas de banano;
Pérez-Pazos y Sánchez-López (2017) evaluaron el efecto de bacterias asociadas al cultivo de batata, con capacidad de promover el crecimiento vegetal en plántulas de batata.

A estos se suman estudios de investigadores de Brasil y México que han realizado aportes relevantes en este campo del conocimiento. A este respecto, Braga et al. (2018) caracterizaron aislados bacterianos obtenidos de las raíces del arroz y determinaron su capacidad para promover el crecimiento de las plantas, mientras que De Gregorio et al. (2017) evaluaron el efecto de inoculantes PGPR en semillas de soja sobre los parámetros de crecimiento. En México por su parte, Morgado González et al. (2015) evaluaron el efecto de cepas PGPR en la variedad comercial de caña; Martínez et al. (2013) identificaron bacterias aisladas de la rizosfera del tomate en función de sus propiedades promotoras del crecimiento vegetal, y evaluaron su efecto en el desarrollo de plántulas de tomate y pimiento.

\section{Géneros microbianos en los PGPR de los estudios incluidos}

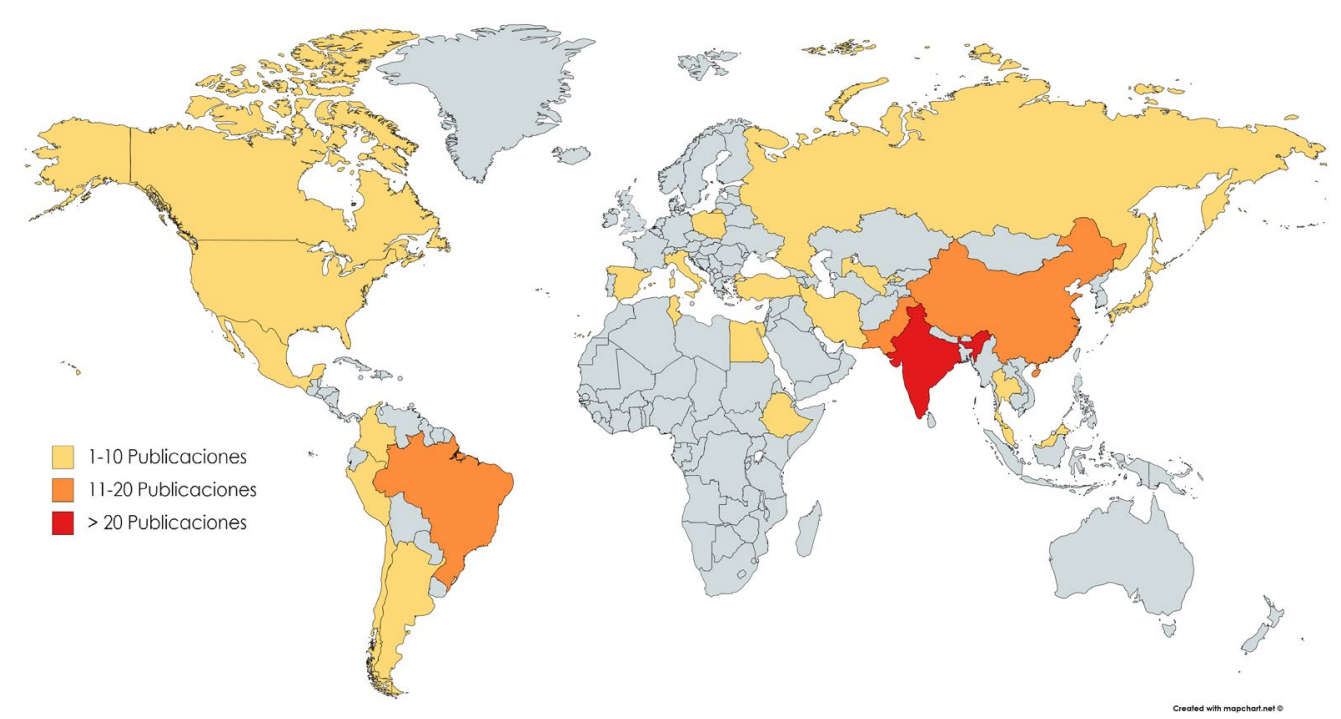

Figura 2. Países de origen de las publicaciones sobre las aplicaciones de las PGPR encontradas en la revisión sistemática.

Fuente: Autores. 
Como resultado de la revisión, se identificaron 63 géneros microbianos diferentes, los más estudiados fueron Bacillus en el $43.1 \%$ (IC95\%=35.1-51.1) de los estudios, Pseudomonas en el $41.5 \%$ (IC95\%=33.3-49.2) y Enterobacter en el $10.6 \%$ (IC95\%=5.5-15.7), en la tabla 1 aparecen los demás géneros identificados en la revisión. Además, los siguientes géneros fueron analizados con una frecuencia de 0.6\% ( $\mathrm{n}=1$ estudio): Agrobacterium, Arthrobacter, Azorhizobium, Bradyrhizobium, Citrobacter, Comamonas, Ensifer, Enterococcus, Exiguobacterium, Fictibacillus, Gluconacetobacter, Gordonia, Hartmannibacter, Lysinibacillus, Lysobacter, Mesorhizobium, Microbacterium, Micrococcus, Mycobacterium, Phosphorobacillus, Planococcus, Promicromonospora, Providencia, Rhodobacter, Rhodococcus, Rhodopseudomonas, Shewanella, Sphingobacterium, Sporosarcina, Streptomyces, Tetrathiobacter, Trabulsiella, Variovorax, Virgibacillus y Viridibacillus. A continuación, se explicitan algunas características importantes de los tres géneros más estudiados en PGPR.

En particular, Pseudomonas es un género con capacidad biocontroladora, responsable de la supresión natural de algunos patógenos transmitidos por el suelo, estimulador del crecimiento de las plantas y solubilizador de minerales; además, presenta una alta tasa de crecimiento en condiciones in vitro, lo cual facilita su producción en masa (Howell y Stipanovic, 1979; Kraus y Loper, 1992; Oteino et al., 2015). Cabe resaltar que Howell y Stipanovic (1980) y Ramamoorthy et al. (2010) pudieron evidenciar que las Pseudomonas fluorescens producen un amplio espectro de metabolitos bioactivos; es decir, antibióticos, sideróforos, volátiles y sustancias promotoras del crecimiento vegetal, permitiéndole una mejor adaptación al estrés ambiental. Lo anterior podría explicar el gran interés como objeto de estudio en diversas investigaciones.
Así mismo, el género Bacillus ha sido ampliamente estudiado por su capacidad para formar endosporas, lo que le confiere resistencia ante condiciones ambientales adversas. Además, posee un gran potencial para estimular el crecimiento vegetal por medio de la síntesis de fitohormonas (ácido indolacético, giberalinas, entre otras), la solubilización de fosfato, y la producción de diferentes metabolitos con actividad antagónica, tales como los lipopéptidos tipo fenginicina y surfactina, que estimulan la resistencia sistémica inducida, e inician la respuesta inmune en las plantas (Glickmann y Dessaux, 1995; Pliego et al., 2011; Xie et al., 2017). Dadas las particularidades metabólicas del género Bacillus y su habilidad para formar endosporas, estas bacterias se presentan como una alternativa altamente promisoria para la formulación de productos biotecnológicos de uso agrícola (Bowen y Rovira, 1999).

Otro género destacable durante la revisión fue Enterobacter, el cual tiene la capacidad de conferir resistencia al estrés salino, ya que posee genes que regulan la homeostasis iónica, facilitando la adaptación de las plantas en condiciones de alta salinidad (Padan, 2008; Sandhya et al., 2010). Las Enterobacter spp. tienen la habilidad de producir fitohormonas (citoquininas, AIA, entre otras), inhibir el crecimiento de fitopatógenos y de solubilizar fosfato, lo cual se ha documentado en diferentes estudios (Mehnaz et al., 2001; Hynes et al., 2008; Oteino et al., 2015). A pesar de las características PGPR dadas por las Enterobacter, se encontró que, en comparación con Bacillus spp. y Pseudomonas spp., fue el género menos reportado en las publicaciones, lo cual se puede deber a que es un género relacionado con infecciones oportunistas de gran importancia clínica, lo cual limita su uso como PGPR (Chart, 2012; Dijun et al., 2016). 
Tabla 1. Distribución porcentual de los géneros microbianos de PGPR estudiados en las publicaciones encontradas en la revisión sistemática.

\begin{tabular}{|c|c|c|c|}
\hline Género & $\mathbf{n}$ & $\%$ & IC 95\% \\
\hline Bacillus & 69 & 43.1 & $35.1-51.1$ \\
\hline Pseudomonas & 66 & 41.3 & $33.3-49.2$ \\
\hline Enterobacter & 17 & 10.6 & $5.5-15.7$ \\
\hline Paenibacillus & 16 & 10.0 & $5.0-15.0$ \\
\hline Azotobacter & 12 & 7.5 & $3.1-12.0$ \\
\hline Burkholderia & 12 & 7.5 & $3.1-12.0$ \\
\hline Serratia & 12 & 7.5 & $3.1-12.0$ \\
\hline Azospirillum & 8 & 5.0 & $1.3-8.7$ \\
\hline Ochromobactrum & 6 & 3.8 & $0.5-7.0$ \\
\hline Klebsiella & 5 & 3.1 & $1.0-7.1$ \\
\hline Pantoea & 5 & 3.1 & $1.0-7.1$ \\
\hline Stenotrophomonas & 5 & 3.1 & $1.0-7.1$ \\
\hline Achromobacter & 4 & 2.5 & $0.7-6.3$ \\
\hline Acinetobacter & 4 & 2.5 & $0.7-6.3$ \\
\hline Brevibacillus & 3 & 1.9 & $0.4-5.4$ \\
\hline Novosphingobium & 3 & 1.9 & $0.4-5.4$ \\
\hline Rhizobium & 3 & 1.9 & $0.4-5.4$ \\
\hline Aeromonas & 2 & 1.3 & $0.2-4.4$ \\
\hline Alcaligenes & 2 & 1.3 & $0.2-4.4$ \\
\hline Aneurinibacillus & 2 & 1.3 & $0.2-4.4$ \\
\hline Cellulosinmicrobium & 2 & 1.3 & $0.2-4.4$ \\
\hline Chryseobacterium & 2 & 1.3 & $0.2-4.4$ \\
\hline Delftia & 2 & 1.3 & $0.2-4.4$ \\
\hline Erwinia & 2 & 1.3 & $0.2-4.4$ \\
\hline Flavobacterium & 2 & 1.3 & $0.2-4.4$ \\
\hline Herbaspirilum & 2 & 1.3 & $0.2-4.4$ \\
\hline Sphingomonas & 2 & 1.3 & $0.2-4.4$ \\
\hline Staphylococcus & 2 & 1.3 & $0.2-4.4$ \\
\hline
\end{tabular}

Fuente: Autores. 


\section{Mecanismos de PGPR en los estudios incluidos}

En total, se identificaron 22 mecanismos PGPR diferentes, siendo AIA (ácido indolacético), solubilización de fosfato, antagonismo y producción de sideróforos los de mayor frecuencia (Tabla 2). Esta evidencia se puede deber a que son mecanismos PGPR que hacen relación tanto a la estimulación del crecimiento como a la actividad biocontroladora, haciendo alusión a la producción de fitohormonas, solubilización de nutrientes e inhibición de fitopatógenos, lo que deriva en efectos benéficos para el desarrollo de las plantas (Glick y Bashan, 1997; Van Loon et al., 1998; Whipps, 2001; Zahir et al., 2003). El AIA, es una de las auxinas más estudiadas dado que produce un incremento en el crecimiento vegetal, además está involucrada en la diferenciación y en la elongación del tallo (Arshad y Frankenberger, 2016). Por otra parte, la solubilización de minerales como el hierro y fosfatos son comúnmente asociados con las PGPR, debido a que facilitan la disponibilidad de estos nutrientes en el suelo y aumentan la productividad de las plantas.

Con respecto a la captación de hierro, esta actividad se encuentra relacionada con el uso de sideróforos bacterianos por las plantas, mientras que la disponibilidad de fosfato está relacionada con la producción de enzimas del grupo de las fosfatasas, lo cual permite un incremento significativo en la toma de este macronutriente, importante para los procesos metabólicos de las plantas (Van Loon et al., 1998; Bakker et al., 2003; Guang-Can et al., 2008; Liu et al., 2015). Aunque se ha demostrado que diferentes géneros bacterianos presentan este rasgo, la mayor parte de los reportes corresponden a Pseudomonas y BaciIlus (Bowen y Rovira, 1999; Zahir et al., 2003).

La capacidad antagónica resulta ser un mecanismo crucial para la defensa de las plantas, ya que permite la inhibición de microorganismos causantes de diversas enfermedades (Glick y Bashan, 1997; Whipps, 2001); además, la evaluación experimental de los mecanismos mencionados con anterioridad, no involucran una alta complejidad a nivel de laboratorio, ni representa un alto costo económico, ya que se emplean metodologías de fácil ejecución (Uzoh et al., 2018).

\section{Especies vegetales en las que se ha estu- diado de efecto de las PGPR}

Dichos mecanismos se evaluaron en 58 especies vegetales diferentes, con mayor frecuencia en maíz, tomate, trigo y arroz (Tabla 3). Además, las siguientes especies se estudiaron en una frecuencia de $0.6 \%(n=1)$ estudios: cañuela, agropiro, alfalfa, lavanda, amapola, arveja, batata, banano, bufera, mungo, calabaza, castor (ricino), cebada, ciruelo, coco, eucalipto, encina, jengibre, kikuyu, kiwi, almorta (Lathyrus), lolium, mango, manzano, margaritas, nabo, sorgo, papa, palma de aceite, tulsí, trebol rojo y timothy. Es posible que se realice con mayor frecuencia esta evaluación en los estudios de las especies maíz, tomate, trigo y arroz, debido a su rápido crecimiento, que permite evaluar el efecto de las PGPR's en las diferentes etapas del crecimiento vegetal. Además, cabe resaltar que son cultivos de gran importancia a nivel mundial, debido a la alta demanda alimentaria (Deshpande, 2017; Agricultural Market Information System (AMIS) 2018). 
Tabla 2. Distribución de frecuencias de los mecanismos PGPR identificados en los estudios descritos en la revisión sistemática.

\begin{tabular}{|c|c|c|c|}
\hline Mecanismos PGPR & $\mathbf{n}$ & $\%$ & IC95\% \\
\hline Ácido indolacético (AIA) & 107 & 66.9 & $59.3-74.5$ \\
\hline Solubilización de fosfato & 97 & 60.6 & $52.7-68.5$ \\
\hline Antagonismo & 63 & 39.4 & $31.5-47.3$ \\
\hline Producción de sideróforos & 58 & 36.3 & $28.5-44.0$ \\
\hline Ácido 1-Aminociclopropano-1 carboxílico (ACC) & 46 & 28.8 & $21.4-36.1$ \\
\hline Ácido cianhídrico (HCN) & 35 & 21.9 & $15.2-28.6$ \\
\hline Fijación de nitrógeno & 27 & 16.9 & $10.8-23.0$ \\
\hline Amoniaco & 12 & 7.5 & $3.1-12.0$ \\
\hline Control biológico & 8 & 5.0 & $1.3-8.7$ \\
\hline Solubilización de zinc & 6 & 3.8 & $0.5-7.0$ \\
\hline Amonio & 5 & 3.1 & $1.0-7.1$ \\
\hline Solubilización de potasio & 5 & 3.1 & $1.0-7.1$ \\
\hline Ácido giberélico & 3 & 1.9 & $0.4-5.4$ \\
\hline Inducción de resistencia sistémica (IRS) & 3 & 1.9 & $0.4-5.4$ \\
\hline Nitrogenasa & 3 & 1.9 & $0.4-5.4$ \\
\hline Ácido salicílico & 2 & 1.3 & $0.2-4.4$ \\
\hline Ácidos orgánicos & 2 & 1.3 & $0.2-4.4$ \\
\hline Giberalinas & 2 & 1.3 & $0.2-44$ \\
\hline ATB (antibióticos) & 1 & 0.6 & $0.0-3.4$ \\
\hline Solubilización de silicio & 1 & 0.6 & $0.0-3.4$ \\
\hline Compuestos orgánicos volátiles (COV) & 1 & 0.6 & $0.0-3.4$ \\
\hline
\end{tabular}

Fuente: Autores. 
Tabla 3. Frecuencia de las especies vegetales en las que se ha estudiado de efecto de las PGPR.

\begin{tabular}{|c|c|c|c|}
\hline Planta estudiada & $\mathbf{n}$ & $\%$ & IC95\% \\
\hline Maíz & 24 & 15.0 & $9.2-20.8$ \\
\hline Tomate & 24 & 15.0 & $9.2-20.8$ \\
\hline Trigo & 13 & 8.1 & $3.6-12.7$ \\
\hline Arroz & 12 & 7.5 & 3.1-11.9 \\
\hline Soja & 9 & 5.6 & $1.7-9.5$ \\
\hline Frijol & 8 & 5.0 & $1.3-8.7$ \\
\hline Garbanzo & 5 & 3.1 & $1.0-7.1$ \\
\hline Canola & 5 & 3.1 & $1.0-7.1$ \\
\hline Chile & 5 & 3.1 & $1.0-7.1$ \\
\hline Pepino & 5 & 3.1 & $1.0-7.1$ \\
\hline Pimienta & 5 & 3.1 & $1.0-7.1$ \\
\hline Maní & 4 & 2.5 & $0.7-6.3$ \\
\hline Caña de azucar & 3 & 1.9 & $0.4-5.4$ \\
\hline Lechuga & 3 & 1.9 & $0.4-5.4$ \\
\hline Mostaza & 3 & 1.9 & $0.4-5.4$ \\
\hline Algodón & 2 & 1.3 & $0.2-4.4$ \\
\hline Berenjena & 2 & 1.3 & $0.2-4.4$ \\
\hline Chicharo salvaje & 2 & 1.3 & $0.2-4.4$ \\
\hline Col & 2 & 1.3 & $0.2-4.4$ \\
\hline Cúrcuma & 2 & 1.3 & $0.2-4.4$ \\
\hline Fenogreco & 2 & 1.3 & $0.2-4.4$ \\
\hline Guisante & 2 & 1.3 & $0.2-4.4$ \\
\hline Remolacha & 2 & 1.3 & $0.2-4.4$ \\
\hline Salvia & 2 & 1.3 & $0.2-4.4$ \\
\hline Sésamo & 2 & 1.3 & $0.2-4.4$ \\
\hline Té & 2 & 1.3 & $0.2-4.4$ \\
\hline
\end{tabular}

Fuente: Autores.

Variables de crecimiento de las plantas usadas en los estudios que demuestran el efecto de las PGPR

Finalmente, en la tabla 4 se presentan las medidas para el evaluar el efecto de las PGPR sobre diferentes componentes de las plantas utilizadas en los estudios, a dichas medidas de eficacia se suman las siguientes, estudiadas con una frecuencia de un (1) estudio: diámetro del brote, diámetro del estoma, diámetro de la raíz, índice de cosecha, longitud del fruto, número de flores, peso de tuzas de mazorcas, peso fresco de 100 vainas, peso fresco total de raíz y brote, peso seco total de raíz y brote, porcentaje de aumento de la raíz, porcentaje de aumento del brote, porcentaje de aumento 
sobre el control, rendimiento foliar, rendimiento por hectárea y tiempo de emergencia.

Además, como se mencionó en el protocolo de búsqueda, se incluyeron artículos en donde se especifica que las condiciones ambientales de las pruebas in vivo no fueron controladas; encontrando que de un total 160 estudios, $71.25 \%$ fueron realizados en invernadero, $17.5 \%$ en campo, $10.62 \%$ incluyeron pruebas en campo e invernadero y un $0.62 \%$ no especifican el sitio de experimento.

Tabla 4. Variables de crecimiento de las plantas usadas en los estudios que demuestran el efecto de las PGPR

\begin{tabular}{|c|c|c|c|}
\hline Variable de respuesta de las plantas & $\mathbf{n}$ & $\%$ & IC95\% \\
\hline Peso seco de la raíz & 70 & 43.8 & $35.8-51.7$ \\
\hline Longitud de raíz & 63 & 39.4 & $31.5-47.3$ \\
\hline Longitud del brote & 63 & 39.4 & $31.5-47.3$ \\
\hline Peso seco del brote & 58 & 36.3 & $28.5-44.0$ \\
\hline Altura de la planta & 48 & 30.0 & $22.6-37.4$ \\
\hline Peso seco de la planta & 43 & 26.9 & $19.7-34.1$ \\
\hline Peso fresco de la raíz & 38 & 23.8 & $16.8-30.7$ \\
\hline Peso fresco de la planta & 33 & 20.6 & $14.0-27.2$ \\
\hline Peso fresco del brote & 28 & 17.5 & $11.3-23.7$ \\
\hline Rendimiento semilla/vaina/paja & 27 & 16.9 & $10.8-23.0$ \\
\hline Área foliar & 15 & 9.4 & $4.5-14.2$ \\
\hline Número de hojas & 14 & 8.8 & $4.1-13.4$ \\
\hline Diámetro del vástago & 12 & 7.5 & $3.1-11.9$ \\
\hline Porcentaje de germinación & 11 & 6.9 & $2.6-111$ \\
\hline Número de raíces principales/laterales & 10 & 6.3 & $2.2-103$ \\
\hline Peso del fruto & 9 & 5.6 & $1.7-9.5$ \\
\hline Número de semillas & 8 & 5.0 & $1.3-8.7$ \\
\hline Índice de vigor & 7 & 4.4 & $0.9-7.9$ \\
\hline Número de nódulos & 7 & 4.4 & $0.9-7.9$ \\
\hline Longitud del vástago & 6 & 3.8 & $0.5-7.0$ \\
\hline Número de frutos & 6 & 3.8 & $0.5-7.0$ \\
\hline Número de vainas & 6 & 3.8 & $0.5-7.0$ \\
\hline Peso fresco de hojas & 6 & 3.8 & $0.5-7.0$ \\
\hline Peso fresco del vástago & 6 & 3.8 & $0.5-7.0$ \\
\hline Peso seco del vástago & 6 & 3.8 & $0.5-7.0$ \\
\hline Número de ramas por planta & 5 & 3.1 & $1.0-7.1$ \\
\hline Número de sierpes & 5 & 3.1 & $1.0-7.1$ \\
\hline Peso de 1000 semillas & 5 & 3.1 & $1.0-7.1$ \\
\hline Producción de fruto/semilla & 5 & 3.1 & $1.0-7.1$ \\
\hline
\end{tabular}




\begin{tabular}{|c|c|c|c|}
\hline Variable de respuesta de las plantas & $\mathbf{n}$ & $\%$ & IC95\% \\
\hline Peso de las semillas & 4 & 2.5 & $0.7-6.3$ \\
\hline Peso de vainas & 4 & 2.5 & $0.7-6.3$ \\
\hline Porcentaje de incremento/raíz/brote/hoja/vaina & 4 & 2.5 & $0.7-6.3$ \\
\hline Rendimiento del fruto & 4 & 2.5 & $0.7-6.3$ \\
\hline Área de la raíz & 3 & 1.9 & $0.4-5.4$ \\
\hline Longitud del brote + raíz & 3 & 1.9 & $0.4-5.4$ \\
\hline Peso de 100 semillas & 3 & 1.9 & $0.4-5.4$ \\
\hline Peso seco de nódulos & 3 & 1.9 & $0.4-5.4$ \\
\hline Rendimiento total de la planta & 3 & 1.9 & $0.4-5.4$ \\
\hline Ancho de la hoja & 2 & 1.3 & $0.2-4.4$ \\
\hline Biomasa del rizoma & 2 & 1.3 & $0.2-4.4$ \\
\hline Diámetro de la mazorca & 2 & 1.3 & $0.2-4.4$ \\
\hline Diámetro del cuello & 2 & 1.3 & $0.2-4.4$ \\
\hline Longitud de la panícula & 2 & 1.3 & $0.2-4.4$ \\
\hline Longitud total de la planta & 2 & 1.3 & $0.2-4.4$ \\
\hline Lontitud de las hojas & 2 & 1.3 & $0.2-4.4$ \\
\hline Número de brotes por planta & 2 & 1.3 & $0.2-4.4$ \\
\hline Número de macollas & 2 & 1.3 & $0.2-4.4$ \\
\hline Número de panículas & 2 & 1.3 & $0.2-4.4$ \\
\hline Peso seco de hojas & 2 & 1.3 & $0.2-4.4$ \\
\hline Porcentaje de aumento del rendimiento & 2 & 1.3 & $0.2-4.4$ \\
\hline Porcentaje de llenado de vainas & 2 & 1.3 & $0.2-4.4$ \\
\hline Relación brote/raíz & 2 & 1.3 & $0.2-4.4$ \\
\hline Rendimiento de la raíz/rizoma & 2 & 1.3 & $0.2-4.4$ \\
\hline
\end{tabular}

Fuente: Autores

En el último año se ha aumentado el número de publicaciones en este tema, destacándose el estudio de Amaresan et al. (2019) donde evaluaron la capacidad de bacterias asociadas a las plantas de tomate para reducir la incidencia de diferentes enfermedades $y$ determinaron sus efectos sobre el rendimiento en condiciones naturales de campo. En su estudio, Dede et al. (2020) evaluaron la actividad antagónica de actinomicetos aislados de la rizosfera del olivo y su efecto promotor de crecimiento con la finalidad de ser utilizados como biofertilizantes en futuros estudios. La investigación de Andy et al. (2020) giró en torno al aislamiento y caracterización de diferentes PGPR de la zona de la raíz de diferentes leguminosas que en el futuro podría resultar como un biofertilizante revolucionario y bioinoculantes.

\section{Recomendaciones para estudios posteriores} Vale la pena señalar que aún se deben realizar investigaciones más exhaustivas que ayuden a la comprensión de los mecanismos de fitoestimulación mediada por las PGPR, que tengan como objetivo detectar cepas de rizobacterias 
más competentes que puedan funcionar en diversas condiciones agroecológicas; esto podría garantizar la estabilidad de las PGPR en la productividad agrícola. Específicamente en los hallazgos obtenidos en esta revisión sistematizada se podrían evaluar en futuros estudios que realicen pruebas de estimulación en campo, determinando la efectividad de las PGPR no solo a nivel de laboratorio sino a gran escala. Además de la evaluación de consorcios de microorganismos efectivos que posteriormente puedan ser utilizados en el desarrollo de formulaciones microbianas, permitiendo de esta manera diseñar y obtener productos agrícolas eficientes, conduciendo como fin último a una agricultura sostenible.

\section{CONCLUSIONES}

En el ámbito mundial, India es el país con más publicaciones sobre PGPR, debido a que es considerado como una potencia agrícola mundial; además de su gran interés por desarrollar biotecnologías en el marco de una agricultura sostenible. Se sintetizó la principal evidencia de los mecanismos PGPR en el ámbito mundial, siendo AIA, solubilización de fosfato, antagonismo y producción de sideróforos los de mayor frecuencia, y abarcan mecanismos directos e indirectos de la forma en que las rizobacterias pueden promover el crecimiento de las plantas. Los microorganismos más estudiados fueron BaciIlus, Pseudomonasy Enterobacter, ya que estos poseen rasgos PGPR efectivos como capacidad biocontroladora, solubilización de minerales, síntesis de fitohomonas y capacidad de conferir resistencia al estrés salino, como es el caso del género Enterobacter. Finalmente, las especies vegetales más frecuentemente estudiadas fueron maíz, tomate, trigo y arroz, cultivos de gran importancia alimentaria. En conjunto, estos hallazgos son relevantes para identificar las principales líneas de acción en futuras investigaciones cuyo propósito sea a mejorar la salud, producción de cultivos y sostenibilidad ambiental.

\section{CONTRIBUCIÓN DE LA AUTORÍA}

Primer autor: metodología, investigación, análisis de datos, conceptualización, escritura - borrador original. Segundo autor: metodología, investigación, análisis de datos, conceptualización, escritura - borrador original. Tercer autor: revisión y edición. Cuarto autor: conceptualización, investigación, logística, escritura - revisión y edición.

\section{AGRADECIMIENTOS}

A los grupos de investigación, Salud y Sostenibilidad y Microbiología Veterinaria de la Escuela de Microbiología, Universidad de Antioquia.

\section{LITERATURA CITADA}

AMIS Agricultural Market Information System. (2018). Amis-Outlook.org. https://app.amisoutlook.org/\#/market-database/supply-and-demand-overview

Amaresan, N., Jayakumar, V., Kumar, K., y Thajuddin, N. (2019). Biocontrol and plant growthpromoting ability of plant-associated bacteria from tomato (Lycopersicum esculentum) under field condition. Microbial Pathogenesis, 136, 103713 https://doi.org/10.1016/j.micpath. 2019.103713

Andy, A., Masih, S. y Gour, V. (2020). Isolation, screening and characterization of plant growth promoting rhizobacteria from rhizospheric soils of selected pulses. Biocatalysis and Agricultural Biotechnology, 27. https://doi.org/10.1016/j. bcab.2020.101685

Arshad, M., y Frankenberger, W.T. (1998). PlantGrowth-Regulating Substances in the Rhizosphere: Microbial Production and Functions. $A d-$ vances in Agronomy, 62, 45-151. https://doi. org/10.1016/S0065-2113(08)60567-2

Braga, L.F., Oliveira, F.A., Couto, E.A., Santos, K.F., Ferreira, E.P., y Martin-Didonet, C.C. (2018). Polyphasic characterization of bacteria obtained from upland rice cultivated in Cerrado soil. Brazilian Journal of Microbiology, 49, 20-28. https:// doi.org/10.1016/j.bjm.2017.04.004

Bakker, P.A.H.M., Ran, L.X., Pieterse, C.M.J., y Van Loon, L.C. (2003). Understanding the involvement of rhizobacteria-mediated induction of systemic resistance in biocontrol of plant diseases. 
Canadian Journal of Plant Pathology, 25, 5-9. https://doi.org/10.1080/07060660309507043

Bowen, G.D., y Rovira, A. D. (1999). The Rhizosphere and Its Management to Improve Plant Growth. Advances in Agronomy, 66, 1-102. https://doi. org/10.1016/S0065-2113(08)60425-3

Chart, H. (2012). Klebsiella, Enterobacter, Proteus and other enterobacteria: Pneumonia, urinary, tract infection; opportunist infection. Medical Microbiology, 8, 290-297. https://doi.org/10.1016/ B978-0-7020-4089-4.00042-1

Centro Cochrane Iberoamericano. (2012). Manual cochrane de revisiones sistemáticas de intervenciones. http://www.cochrane.es/?q=es/ node/269

Dede, A., Güven K., y Şahi N.N. (2020), Isolation, plant growth-promoting traits, antagonistic effects on clinical y plant pathogenic organisms and identification of actinomycetes from olive rhizosphere. Microbial Pathogenesis, 143,104-134. https://doi.org/10.1016/j.micpath.2020.104134

De Gregorio, P. R., Michavila, G., Ricciardi Muller, L., de Souza Borges, C., Pomares, M. F., Saccol de Sá, E. L., Pereira, C., y Vincent, P. A. (2017). Beneficial rhizobacteria immobilized in nanofibers for potential application as soybean seed bioinoculants. PLOS ONE, 12(5), e0176930. https://doi. org/10.1371/journal.pone.0176930

Deshpande, T. (2017). State of Agriculture. PRS Legislative Research. https://prsindia.org/policy/ analytical-reports/state-agriculture-india

Dijun, Z., Weina, H., Qianqian, T., Jun, Z., y Xiurong., S. (2016). Multi-omics analysis in the pathogenicity of Enterobacter cloacea ENHKU01 isolated from swage outfalls along the Ningbo coastline. Proteome Science 14, 1-15. https:// doi.org/10.1186/s12953-016-0104-y

Etesami, H., y Maheshwari, D.K. (2018). Use of plant growth promoting rhizobacteria (PGPRs) with multiple plant growth promoting traits in stress agriculture: action mechanisms and future prospects. Ecotoxicology and Environmental safety, 156, 225-246. https://doi.org/10.1016/j. ecoenv.2018.03.013

Glick, B.R., y Bashan, Y. (1997). Genetic manipulation of plant growth-promoting bacteria to enhance biocontrol of phytopathogens. Biotechnology Advances, 15, 353-378. https://doi. org/10.1016/S0734-9750(97)00004-9
Glickmann, E., y Y., Dessaux. (1995). A Critical Examination of the Specificity of the Salkowski Reagent for Indolic Compounds Produced by Phytopathogenic Bacteria. Applied and Environmental Microbiology, 61, 793-96. https://doi. org/10.1128/AEM.61.2.793-796.1995

Guang-Can, T., Shu-Jun, T., Miao-Ying, C., y GuangHui, X. (2008). Phosphate-Solubilizing and -Mineralizing Abilities of Bacteria Isolated from Soils. Pedosphere, 18(4), 515-523. https:// doi.org/10.1016/S1002-0160(08)60042-9

Howell, C., y Stipanovic, R. (1979). Control of Rhizoctonia solani on cotton seedlings with Pseudomonas fluorescens and with an antibiotic produced by the bacterium. Phytopathology, 69, 480-482. https://doi.org/10.1094/Phyto-69-480

Howell, C., y Stipanovic, R. (1980). Suppression of Pythium ultimium induced damping off of cotton seedlings by Pseudomonas fluorescens and its antibiotic pyoluterin. Phytopathology, 70,712715. https://doi.org/10.1094/Phyto-70-712

Hynes, R.K., Leung, G.C., Hirkala, D.L., y Nelson, L.M. (2008). Isolation, selection, and characterization of beneficial rhizobacteria from pea, lentil, and chickpea grown in western Canada. Canadian Journal Microbiology, 54(4), 248-258. https://doi.org/10.1139/W08-008

Jewell, M.C., Campbell, B.C., y Godwin, I.D. (2010). Transgenic Plants for Abiotic Stress Resistance. Transgenic Crop Plants. https://do..org/10.1007/ 978-3-642-04812-8_2

Karthiba, L., Saveetha, K., Suresh, S., Raguchander, T., Saravanakumar, D., y Samiyappan, R. (2010). PGPR and entomopathogenic fungus bioformulation for the synchronous management of leaffolder pest and sheath blight disease of rice. Pest Management Science, 66(5), 555-564. https://doi.org/10.1002/ps.1907

Kloepper, J.W. (1993). Plant growth-promoting rhizobacteria as biological control agents. Soil Microbial Ecology: Applications in agricultural and environmental Management. Plant growth-promoting rhizobacteria as biological control agents (Metting, F.B., Jr. Ed., pp. 255-274). Marcel Dekker, Inc.

Kraus, J., y Loper, J. (1992). Lack of evidence for a role of antifungal metabolite production by Pseudomonas fluorescens Pf-5 in biological control of Pythium damping-off of cucumber. Molecular Plant Pathology, 82(3), 264-271. https:// doi.org/10.1094/Phyto-82-264 
Lavakush, Yadav, J., Verma, J. P., Jaiswal, D. K., y Kumar, A. (2014). Evaluation of PGPR and different concentration of phosphorus level on plant growth, yield and nutrient content of rice (Oryza sativa). Ecological Engineering, 62, 123-128. https://doi.org/10.1016/j.ecoleng.2013.10.013

Liu, Z., Li, Y. C., Zhang, S., Fu, Y., Fan, X., Patel, J. S., y Zhang, M. (2015). Characterization of phosphate-solubilizing bacteria isolated from calcareous soils. Applied Soil Ecology 96, 217224. https://doi.org/10.1016/j.apsoil.2015.08.003

Martínez, L.L, Martínez Peniche, R.A., Hernández Iturriaga, M., Arvizu Medrano, S.M., y Pacheco Aguilar, J.R. (2013). Caracterización de rizobacterias aisladas de tomate y su efecto en el crecimiento de tomate y pimiento. Revista fitotecnia mexicana, 36(1), 63-69. Recuperado de http://www.scielo.org.mx/scielo.php?script=sci_ arttext\&pid=S0187-73802013000100007\&Ing=es \&tlng=es

Mahmood, S., Daur, I., Al-Solaimani, S.G., Ahmad, S., Madkour, M.H., Yasir, M., Hirt, H., Ali, S., y Ali, Z. (2016). Plant growth promoting rhizobacteria and silicon synergistically enhance salinity tolerance of mung bean. Frontiers in Plant Science, 7, 876. https://doi.org/10.3389/fpls.2016.00876

Mehnaz, S., Mirza, M.S., Haurat, J., Bally, R., Normand, P., Bano, A., y Malik, K.A. (2001). Isolation and $16 S$ rRNA sequence analysis of the beneficial bacteria from the rhizosphere of rice. Canadian Journal of Microbiology, 47(2), 110-117. https:// doi.org/10.1139/w00-132

Mhatre, P. H., Karthik, C., Kadirvelu, K., Divya, K. L., Venkatasalam, E. P., Srinivasan, S., Ramkumar, G., Saranya, C., y Shanmuganathan, R. (2018). Plant Growth Promoting Rhizobacteria (PGPR): A potential alternative tool for Nematodes biocontrol. International Society of Biocatalysis and Agricultural Biotechnology, 17, 119-128. https:// doi.org/10.1016/j.bcab.2018.11.009

Morgado González, A., Espinosa Victoria, D., y Gómez Merino, F.C. (2015). Efficiency of plant growth promoting rhizobacteria (PGPR) in sugarcane. Terra Latinoamericana, 33(4), 321330. Recuperado de http://www.scielo.org. $\mathrm{mx} /$ scielo.php?script $=$ sci_arttext\&pid $=$ S018757792015000400321\&lng=es\&tIng=

Mukhtar, S., Shahid, I., Mehnaz, S., y Malik, K. (2017). Assessment of two carrier materials for phosphate solubilizing biofertilizers and their effect on growth of wheat (Triticum aestivum L.).
Microbiological Research, 205, 107-117. https:// doi.org/10.1016/j.micres.2017.08.011

Oteino, N., Lally, R.D., Kiwanuka, S., Lloyd, A., Ryan, D., Germaine, K.J., y Dowling, D.N. (2015). Plant growth promotion induced by phosphate solubilizing endophytic Pseudomonas isolates. Frontiers in Microbiology, 6, 745-754. https://doi.org/10.3 389/fmicb.2015.00745

Padan, E. (2008). The enlightening encounter between structure and function in the $\mathrm{NhaA} \mathrm{Na+-H+}$ antiporter. Trends in Biochemical Sciences, 33(9), 435-443. https://doi.org/10.1016/j.tibs.2008.06.

$\underline{007}$

Pérez-Pazos, J.V y Sánchez-López, D.B. (2017). Caracterización y efecto de Azotobacter, Azospirillum y Pseudomonas asociadas a Ipomoea Batatas del Caribe Colombiano. Revista Colombiana de Biotecnología, 19(2), 35-46. https:// doi.org/10.15446/rev.colomb.biote.v19n2.69471

Pliego, C., Lugtenberg, B., y Kamilova, F. (2011). Plant Growth-Promoting Bacteria: Bacteria: Fundamentals and Exploitation en D.K. Maheswari (Ed). Bacteria in Agrobiology: Crop Ecosystems (pp. 295-344). Springer.

Posada, L., Ramírez, M., Ochoa-Gómez, N., CuellarGaviria, T.Z., Argel-Roldan, L.E., Ramírez, C., y Villegas-Escobar, V. (2016). Bioprospecting of aerobic endospore-forming bacteria with biotechnological potential for growth promotion of banana plants. Scientia Horticulturae, 212, 81-90. https://doi.org/10.1016/j.scienta.2016.09.040

Ramamoorthy, V., Viswanathan, R., Raguchander, T., Prakasam, V., y Samiyappan, R. (2001). Induction of systemic resistance by plant growth promoting rhizobacteria in crop plants against pests and diseases. Crop Protection, 20(1), 1-11. https://doi.org/10.1016/S0261-2194(00)00056-9

Rizvi, A., y Khan, M.S., (2018). Heavy metal induced oxidative damage and root morphology alterations of maize (Zea mays L.) plants and stress mitigation by metal tolerant nitrogen fixing Azotobacter chroococcum. Ecotoxicology and Environmental safety, 157, 9-20. https:// doi.org/10.1016/j.ecoenv.2018.03.063

Sandhya, V., Ali, S.Z., Grover, M., Reddy, G., y Venkateswarlu, B. (2010). Effect of plant growth promoting Pseudomonas spp. on compatible solutes, antioxidant status and plant growth of maize under drought stress. Plant Growth Regulation, 62(1), 21-30. https://doi.org/10.1007/s10 725-010-9479-4 
Schroth, M.N. y Becker, J.O. (1990). Biological control of soil-borne plant pathogens [Presentación en papel, pp. 389-414]. $5^{\text {th }}$ Internacional Congress of Plant Pathology, Kyoto, Japan.

Urrutia, G., y Bonfill, X. (2010). Declaración PRISMA: Una propuesta para mejorar la publicación de revisiones sistemáticas y metaanálisis. Medicina Clinica, 135(11), 507-511.

Uzoh, I. M., y Babalola, O.O. (2018). Rhizosphere biodiversity as a premise for application in bio-economy. Agriculture, Ecosystems \& Environment, 265, 524-534. https://doi.org/10.10 $16 /$ j.agee.2018.07.003

Van Loon, L.C, Baker, P.A.H.M., y Pieterse, C.M.J. (1998). Systemic resistance induced by rhizosphere bacteria. Annual Rewiew of Phytopathology, 36(1), 453-483. https://doi.org/10.1146/ann urev. phyto.36.1.453

Wezel, A., y Soldat, V. (2009). A quantitative and qualitative historical analysis of the scientific discipline of agroecology. International Journal of Agricultural Sustainability, 7, 3-18. https:// doi.org/10.3763/ijas.2009.0400

Whipps, J.M. (2001). Microbial interactions and biocontrol in the rhizosphere. Journal of Experimental Botany, 52, 487-511. https://doi.org/10.109 3/jexbot/52.suppl 1.487
World Bank. (2012). India: Issues and Priorities for Agriculture. https://www.worldbank.org/en/ news/feature/2012/05/17/india-agriculture-issues-priorities

Xiang, N., Lawrence, K.S., Kloepper, J.W., Donald, P.A., y McInroy, J.A. (2017). Biological control of Heterodera glycines by spore-forming plant growth-promoting rhizobacteria (PGPR) on soybean. PLOS ONE, 12(7). https://doi.org/10.1 371/journal.pone.0181201

Xie, Y., Wu, L., Zhu, B., Wu, H., Gu, Q., Rajer, F.U., y Gao, X. (2017). Digital gene expression profiling of the pathogen-resistance mechanism of Oryza sativa 9311 in response to Bacillus amyloliquefaciens FZB42 induction. Biological Control, 110, 89-97. https://doi.org/10.1016/j.bi ocontrol.2017.04.009

Zahir, Z., Arshad, M., y Frankenberger, W.T. (2003). Plant growth-promoting rhizobacteria: applications and perspectives in agriculture. Advances in Agronomy, 81, 97-168. https://doi.org/10.1 016/S0065-2113(03)81003-9
Conflicto de Intereses Los autores declaran no tener ningún conflicto de intereses 